02

\title{
Поверхностно-радиационные моды и продольные экситоны в спектрах экситон-поляритонной люминесценции
}

\author{
() Б.Ж. Ахмадалиев, Н.Х. Юлдашев, И.И. Юлчиев \\ Ферганский политехнический институт, \\ 150107 Фергана, Узбекистан \\ e-mail: uzferfizika@mail.ru
}

Поступила в редакцию 20.02.2018г.

Исследованы низкотемпературные $(T=2 \mathrm{~K})$ спектры экситон-поляритонной люминесценции в окрестности частоты экситонного резонанса $A_{n=1}$ кристаллов $\mathrm{CdS}$ с учетом затухания $\hbar$ механических экситонов. Проанализированы результаты численных расчетов парциальных и интерференционных вкладов объемных и поверхностно-радиационных спектральных мод в геометрии излучения добавочных волн в вакуум с $s$ - и p-поляризациями. Показано, что вклады чисто продольных экситонов и их интерференции с поляритонами верхней дисперсионной ветви в люминесценцию вблизи продольной частоты $\omega_{L}$ небольшие $(\sim 10-30 \%)$. Тем не менее, учет их необходим для получения количественного согласия с экспериментом и именно они обусловливают формирование дополнительной линии $A_{L}^{\prime}$ при наклонном излучении.

DOI: $10.21883 /$ OS.2018.09.46546.52-18

\section{Введение}

Поляритонный механизм люминесценции играет важную роль в формировании низкотемпературных спектров излучения полупроводниковых кристаллов в окрестности экситонных резонансов. К настоящему времени достигнуто понимание многих качественных сторон явления поляритонной люминесценции (ПЛ), связанных с особенностями дисперсии поляритонов, условиями прохождения излучения через границу в области резонанса, характером пространственного и энергетического распределения поляритонов [1-9]. Детальное изучение экситонной энергетической структуры кристаллов показывает, что в формировании спектров низкотемпературной фотолюминесценции наряду с явлениями светоэкситонного взаимодействия (поляритонный эффект) и пространственной дисперсии (зависимость тензора диэлектрической проницаемости $\varepsilon_{\alpha \beta}$ от волнового вектора $\mathbf{k}$ ) существенную роль могут играть эффекты, связанные с экситонным затуханием $\hbar$. В частности, экситонное затухание может обусловливать возгорание излучения поверхностно-радиационных мод (чисто пространственно затухающих волн вглубь кристалла при $\Gamma=0)$ и интерференционной люминесценции когерентно излучающих состояний различных дисперсионных ветвей с близкими значениями $\omega$ и $\mathbf{k}$. В работах [6-9] была построена теория ПЛ в случае, когда диссипативное затухание экситона нарушает критерии применимости кинетического уравнения Больцмана

$$
\left|\operatorname{Rek}_{\beta}\right| \gg \alpha_{\beta},
$$

и справедливая при произвольном соотношении между $\left|\operatorname{Rek}_{2}\right|$ и $\alpha_{2}$ (где $\mathbf{k}_{2}$ и $\alpha_{2}-$ волновой вектор и коэффициент поглощения поляритонов верхней дисперсионной ветви 2). На основе этой теории были последовательно рассчитаны вклады волн нижней поляритонной ветви 1 и сильно затухающей волны 2 в ПЛ в окрестности частоты $\omega_{L}$ продольного экситона (ветвь 3) и удовлетворительно проанализированы экспериментальные спектры ПЛ кристаллов $\mathrm{ZnP}_{2}, \mathrm{CdS}$ и CdTe. Однако при этом конкретный расчет и эксперимент ограничивались самым простым случаем - анализом нормально выходящего из кристалла в вакуум поляритонного излучения, когда отсутствует вклад продольных экситонов в ПЛ. Хотя уже давно известно [3-5], что при наличии пространственной дисперсии (ПД) возможен перенос энергии в кристаллах чисто продольными экситонами и они проявляются в спектрах низкотемпературной фотолюминесценции кристаллов $\mathrm{CdS}$ лишь при наклонном излучении $[7,8]$. Тем не менее, количественный анализ их вкладов в экситон-поляритонную люминесценцию (ЭПЛ) до сих пор не проводился.

В настоящей работе обобщается микроскопическая теория ПЛ кристаллов с ПД типа $\mathrm{CdS}$ с учетом вкладов излучения чисто продольных экситонов в окрестности частоты $\omega_{L}$. Рассматривается ЭПЛ в случае, когда неравенство (1) одновременно нарушается для добавочных волн $\beta=2$ и $\beta=3$, но остается справедливым для поляритонов нижней дисперсионной ветви $\beta=1$. Развитая теория ЭПЛ вопреки обычной теории ПЛ, основанной на использовании функции распределения поляритонов, позволяет учесть интерференцию когерентно излучающих состояний поперечных поляритонов и продольных экситонов при наличии затухания $\Gamma \neq 0$ (что несомненно является новым эффектом, отсутствовавшим в работах $[6,7,9-11])$, т. е. выйти за пределы применимости кинетического уравнения для волн 2 и 3, определяемым наряду с (1) также и более жестким условием

$$
\left|\operatorname{Re}\left(\mathbf{k}_{\beta}-\mathbf{k}_{\beta^{\prime}}\right)\right| \gg \alpha_{\beta}, \alpha_{\beta^{\prime}} \quad\left(\beta, \beta^{\prime}=2,3\right) .
$$


При выполнении неравенства (2) можно пренебречь интерференционным вкладом когерентно излучающих поляритонных состояний разных дисперсионных ветвей. С помощью этой теории здесь проводится анализ угловой зависимости экспериментальных спектров ЭПЛ кристаллов $\mathrm{CdS}$ с гексагональной точечной симметрией для $s$ - и $p$-поляризаций излучения.

\section{Теория}

Рассмотрим дипольно-активный триплетный экситон, характеризуемый резонансной частотой $\omega_{0}$, эффективной массой $M$ и продольно-поперечным расщеплением $\omega_{L T}=\omega_{L}-\omega_{0}$ в кристалле с изотропным тензором диэлектрической проницаемости $[5,6,9]$ :

$$
\begin{aligned}
\varepsilon_{\alpha, \beta}(\omega \mathbf{k}) & =\varepsilon(\omega, \mathbf{k}) \delta_{\alpha \beta} \\
& =\varepsilon_{b}\left[1+\frac{\omega_{L T}}{\omega_{0}(\mathbf{k})-\omega-i \Gamma(\omega, \mathbf{k}) / 2}\right] \delta_{\alpha \beta},
\end{aligned}
$$

где $\varepsilon_{b}-$ фоновая диэлектрическая проницаемость, $\hbar \omega_{0}(\mathbf{k})=\hbar \omega_{0}+\frac{\hbar^{2} \mathbf{k}^{2}}{2 M}-$ энергия механического экситона, $\Gamma(\omega, \mathbf{k})$ - затухание экситона, определяемое процессами рассеяния или захвата. Известно, что в таких кристаллах могут возбуждаться нормальные волны двух типов - поперечные $\left(\varepsilon(\omega, \mathbf{k})=c^{2} k^{2} / \omega^{2}\right.$, где $c-$ скорость света в вакууме) и продольные $(\varepsilon(\omega, \mathbf{k})=0)$. Для большинства полупроводников $\omega_{L T} \ll \omega_{0}$ (например, для $\mathrm{CdS} \omega_{L T}=2.0 \mathrm{meV}, \omega_{0}=2552.4 \mathrm{meV}$ при $\left.T=2 \mathrm{~K}\right)$ и в окрестности резонанса $\omega_{0}$, определяемой условием

$$
\left|\omega-\omega_{0}\right| \ll \sqrt{\omega_{0} \omega_{L T}},
$$

дисперсионные уравнения поперечных поляритонов $\beta=1,2$ и продольных экситонов $\beta=3$ можно представить в упрощенном виде:

$$
\begin{aligned}
& \omega=\omega_{\mathbf{k}}^{(T)}=\omega_{0}+\frac{\hbar \mathbf{k}^{2}}{2 M}-\frac{\varepsilon_{b} \omega_{L T}}{\left(c \mathbf{k} / \omega_{0}\right)^{2}-\varepsilon_{b}}-i \frac{\Gamma(\omega, \mathbf{k})}{2}, \\
& \omega=\omega_{\mathbf{k}}^{(3)}=\omega_{L}+\frac{\hbar \mathbf{k}^{2}}{2 M}-i \frac{\Gamma(\omega, \mathbf{k})}{2}, \omega_{L}=\omega_{0}+\omega_{L T},
\end{aligned}
$$

причем поляритоны 1 и 2 описываются формулой (5) соответственно в областях $k>k_{0} \sqrt{\varepsilon_{b}}$ и $k<k_{0} \sqrt{\varepsilon_{b}}$, где $k_{0}=\omega_{0} / c$.

C ростом затухания $\hbar \Gamma$ дисперсионные кривые, согласно (5) и (6), сильно изменяются. Это особенно заметно вблизи продольной частоты $\omega_{L}$ для мод 2 и 3 , которые при $\hbar \Gamma=0$ являются распространяющимися лишь в области частот $\omega>\omega_{\theta}$ и чисто затухающими, т. е. поверхностно-радиационными, когда $\omega<\omega_{\theta}$. Характерная частота $\omega_{\theta} \geq \omega_{L}$ определяется условием полного внутреннего отражения волн $\beta=2,3$ :

$$
\operatorname{Re} n_{\beta}=c \operatorname{Re} k_{\beta}(\omega) / \omega=\sin \theta .
$$

Здесь $\theta$ - угол выхода излучения из кристалла в вакуум, $n_{\beta}$ - показатель преломления кристалла для волны $\beta$, причем $\operatorname{Re} n_{\beta}<1$ при $\omega<\omega_{\theta}$. Заметим, что, во-первых, появление при $\hbar \Gamma \neq 0$ мнимой части волнового вектора $\mathbf{k}_{\beta}$ при $\omega>\omega_{\beta}$ означает определенное пространственное затухание распространяющихся мод, что приводит в некоторой области частот $\omega-\omega_{\theta} \sim \Gamma$ к нарушению неравенств (1) и (2). Во-вторых, в области частот $\omega<\omega_{\theta}$, где всегда нарушается условие (1) для волн 2 и 3, при конечном Г возникает вещественная добавка к волновому вектору $\mathbf{k}_{\beta}$, которая означает, что поверхностно-радиационные моды 2 и 3 перестают быть чисто затухающими и включаются в перенос энергии экситонного возбуждения в кристалле. Последние обстоятельства безусловно имеют существенное значение в формировании спектра ЭПЛ кристаллов в геометрии излучения чисто продольных экситонов, исследование которых и является основной целью настоящей работы.

Исследуем спектральную интенсивность излучения $I_{\lambda}^{(0)}(\omega, \Omega)$ с поляризацией $\lambda$, распространяющегося в вакууме у поверхности кристалла $(z=-0)$ в направлении единичного вектора $\Omega(\theta, \varphi)$ (который лежит в плоскости $y z$, а внутренняя нормаль к границе кристалла направлена вдоль оси $z ; \lambda=s, p$, где $s\|x, p\| y)$ :

$$
I_{\lambda}^{(0)}(\omega, \Omega)=\sum_{\beta} I_{\beta \lambda}^{(0)}(\omega, \Omega),
$$

$\beta=1,2$ при $\lambda=s$ и $\beta=1,2,3,23$ при $\lambda=p$. Парциальная интенсивность $I_{23 p}^{(0)}$ описывает интерференционный вклад в ЭПЛ волн 2 и 3. Считаем, что в окрестности частоты $\omega_{L}$ можно пренебречь рассеянием волн внутри ветвей $\beta=2,3$ и не учитываем генерацию волн 2 и 3 с малыми значениями $\mathbf{k}$, происходящую за счет рассеяния на LO-фононах из вышележащих экситонных состояний, а также возможное их индуцирование многократным зеркальным отражением волн 1 от внутренней границы кристалла [5]. Таким образом, функция распределения $f$ поляритонов в основном формируется за счет их релаксации по состояниям поперечной моды $1-f_{1 \mathrm{k} \lambda}(\omega, z)$.

Последовательное определение функции $f_{1 \mathbf{k} \lambda}(\omega, z)$ выходит за рамки настоящей работы, и она считается заданной. Естественно, что в кубическом кристалле в результате многократных рассеяний происходит изотропизация функции распределения по направлениям и состояниям поляризации. Далее при расчете в узкой области частот вблизи $\omega_{L}$ достаточно ограничится простейшей факторизованной формой

$$
f_{1 \mathbf{k} \lambda}(\omega, z)=f_{1}(\omega) \exp (-z / L)
$$

содержащей некоторую плавную частотную зависимость $f_{1}(\omega)$. Здесь $L-$ эффективная глубина распределения поляритонов ветви 1, определяемая процессами многократного рассеяния экситон-поляритонов. Координатная зависимость $f_{1 \mathbf{k} \lambda}(\omega, z)$ только от $z$ соответствует однородности задачи вдоль поверхности. 
$\beta \mathbf{k} \lambda$

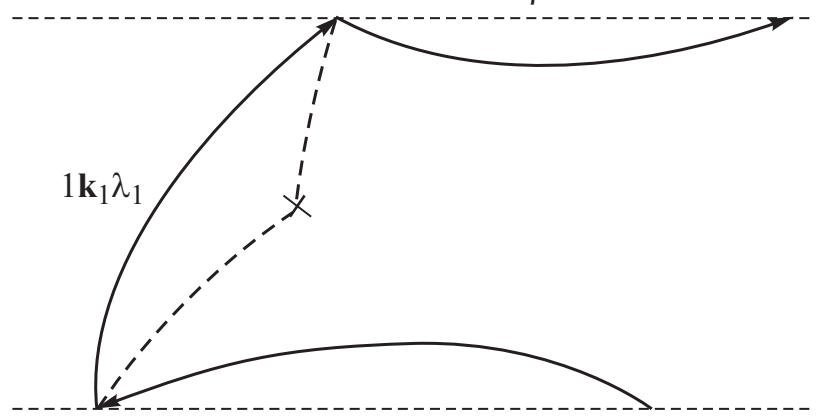

$\beta^{\prime} \mathbf{k}^{\prime} \lambda^{\prime}$

Рис. 1. Диаграмма для функции Грина $G_{\beta \lambda}^{-+}\left(\mathbf{k}, \mathbf{k}^{\prime} ; \omega\right)$. При $\lambda=s$ индекс $\beta=\beta^{\prime}=2$, при $\lambda=p-\beta, \beta^{\prime}=2,3$.

Парциальный вклад $I_{1 \lambda}^{(0)}$ определяется по формуле [5]:

$$
I_{1 \lambda}^{(0)}(\omega, \Omega)=\frac{k_{0}^{2}}{(2 \pi)^{3}} \hbar \omega T_{01}^{(\lambda)} f_{1 \mathbf{k} \lambda}(z=+0),
$$

где

$$
T_{0 \beta}^{(\lambda)}=\frac{I_{N}^{(0)}}{I_{N}^{(\beta)}}=\frac{\cos \theta}{\cos \theta_{\beta}} \frac{1}{n_{\beta}} \frac{w_{0}}{w_{\beta}}\left|t_{0 \beta}^{(\lambda)}\right|
$$

- энергетический коэффициент пропускания волны $\beta$ с поляризацией $\lambda$ из кристалла в вакуум, $t_{0 \beta}^{(\lambda)}-$ соответствующий амплитудный коэффициент пропускания, $I_{N}$ - нормальная составляющая плотности потока энергии к поверхности, $n_{\beta} w_{\beta}-$ коэффициент связи между плотностью потока энергии и квадратом модуля амплитуды электрического поля нормальной волны $\beta$ :

$$
\begin{gathered}
w_{0}=\frac{c}{8 \pi}, w_{\beta}=\frac{c}{8 \pi}\left[1-\delta_{\beta 3}+\frac{\omega_{M} \varepsilon_{b}}{\omega_{L T}}\left(\frac{n_{\beta}^{2}}{\varepsilon_{b}}-1+\delta_{\beta 3}\right)^{2}\right], \\
\beta=1-3 .
\end{gathered}
$$

Интенсивность ЭПЛ с $s$-поляризацией

$$
I_{s}^{(0)}(\omega, \Omega)=I_{1 s}^{(0)}(\omega, \Omega)+I_{2 s}^{(0)}(\omega, \Omega)
$$

не содержит интерференционный член, так как условие (2) считаем выполненным. Парциальный вклад $I_{1 s}^{(0)}$ определяется в кинетическом приближении формулой (8) с $\lambda=s$. Для расчета $I_{2 s}^{(0)}$ при конечных значениях экситонного затухания, т.е. с учетом произвольного соотношения между $\operatorname{Re} k_{2}$ и $\alpha_{2}$, воспользуемся диаграммной техникой Келдыша и рассчитаем функцию Грина $G_{2 s}^{-+}\left(\mathbf{k}, \mathbf{k}^{\prime} ; \omega\right)$ для поляритонов ветви 2 с поляризацией $\lambda$ при заданной функции распределения поляритонов ветви 1 в пренебрежении рассеянием внутри ветви 2. На рис. 1 представлена диаграмма для функции $G_{\beta \lambda}^{-+}\left(\mathbf{k}, \mathbf{k}^{\prime} ; \omega\right)$. Сплошным линиям отвечают функции
Грина экситонов, перенормированные с учетом экситонфотонного и экситон-решеточного взаимодействия:

$$
\begin{gathered}
G_{\mathbf{k} \omega}^{-+}=\left[\omega-\omega_{\mathbf{k}}^{(T)}+i \Gamma(\omega, \mathbf{k}) / 2\right]^{-1}, G_{\mathbf{k} \omega}^{++}=-\left(G_{\mathbf{k} \omega}^{--}\right)^{*}, \\
G_{1 \mathbf{k} \lambda \omega}^{-+}(z)=2 \pi f_{1 \mathbf{k} \lambda}(z) \delta\left(\omega-\omega_{1 \mathbf{k}}^{(T)}\right),
\end{gathered}
$$

где $\Gamma(\omega, \mathbf{k})$ - затухание поляритона, определяемое процессами рассеяния или захвата. Функция $G_{\mathbf{k} \omega}^{--}\left(G_{\mathbf{k} \omega}^{++}\right)$ разлагается на сумму двух полюсных слагаемых:

$G_{\mathbf{k} \omega}^{--}=\sum_{\beta=1,2} G_{\beta \mathbf{k} \omega}^{--}, \quad G_{\beta \mathbf{k} \omega}^{--}=(-1)^{\beta} \frac{2 M}{\hbar} \frac{k_{\beta}^{2}-\varepsilon_{b} k_{0}^{2}}{k_{1}^{2}-k_{2}^{2}} \frac{1}{k^{2}-k_{\beta}^{2}}$.

При расчете $I_{2 s}^{(0)}$ верхней и нижней экситонным линиям на рис. 1 сопоставляются функции $G_{2 \mathbf{k} \omega}^{--}$и $G_{2 \mathbf{k}^{\prime} \omega}^{++}$. Учитывать диаграмму с внешними линиями $G_{1 \mathbf{k} \omega}^{-\frac{2}{2} \omega} G_{1 \mathbf{k} \omega}^{++}$, т.е. учитывать рассеяние $1 \rightarrow 1$ не требуется, так как функция $f_{1 \mathrm{k} \lambda}(z)$ считается заданной. Используя правила диаграммной техники, получим

$$
\begin{aligned}
& I_{2 s}^{(0)}(\omega, \Omega)=\frac{1}{8 \pi} \frac{k_{0}^{2}}{(2 \pi)^{3}}\left|t_{02}^{(s)}\right|^{2}\left\langle\left|E_{s}^{(2)}\left(\mathbf{k}_{\perp}, \omega ; z=+0\right)\right|^{2}\right\rangle, \\
& \left\langle\left|E_{s}^{(2)}\left(\mathbf{k}_{\perp}, \omega ; z\right)\right|^{2}\right\rangle=C \sum_{\mathbf{k}_{1} \lambda_{1}} \sum_{j} \mid \sum_{k_{z}} \frac{G_{2 \mathbf{k} \omega}^{--}}{n_{2}^{2}(\omega)-\varepsilon_{b}} \\
& \quad \times\left.\exp \left[i k_{z}\left(z-z_{j}\right)\right] e_{s}^{\left(\mathbf{k}_{1} \lambda_{1}\right)} V\left(\mathbf{k}_{1}-\mathbf{k}_{2}\right)\right|^{2} G_{1 \mathbf{k}_{1} \lambda_{1} \omega}^{-+}(z) .
\end{aligned}
$$

Здесь $E_{s}^{(2)}\left(\mathbf{k}_{\perp}, \omega ; z\right)$ - амплитуда световой волны с частотой $\omega$ и поперечной составляющей $\mathbf{k}_{\perp}$ (отметим, что $\mathbf{k}_{\perp}$ является хорошим квантовым числом), равной поперечной составляющей $k_{0} \Omega_{\perp}$ волнового вектора этой волны в вакууме, $t_{02}^{(s)}$ - амплитудный коэффициент пропускания, $n_{2}=k_{2} / k_{0}-$ показатель преломления волны $2, e^{\left(\mathbf{k}_{1} \lambda_{1}\right)}-$ вектор поляризации, $e_{s}^{\left(\mathbf{k}_{1} \lambda_{1}\right)}-$ его проекция на ось $X, z_{j}$ - координата $j$-го рассеивающего центра, $C$ - константа.

Подставляя (9b) в (9a), заменяя суммирование по $j$ интегрированием по $\mathbf{r}_{j}$ согласно правилу $\sum_{j} \rightarrow N_{i} \int d \mathbf{r}_{j}$ и интегрируя по $k_{z}$ и $\mathbf{k}_{1}$, окончательно получим

$$
I_{2 s}^{(0)}(\omega, \Omega)=\frac{\left|t_{02}^{(s)}\right|^{2}}{(2 \pi)^{3}} \frac{f_{1}(\omega)}{2 k_{0} \operatorname{Im} n_{2 z}+L^{-1}} \frac{2 M^{2} c \varepsilon_{b} \omega_{L T} \cos \theta}{\hbar \tau_{21}\left|n_{2 z}\left(n_{1}^{2}-n_{2}^{2}\right)\right|^{2}},
$$

где $n_{2 z}=\left(n_{2}^{2}-\sin ^{2} \theta\right)^{1 / 2}$ величины $t_{02}^{(s)}$ и $n_{2}$ рассчитываются с учетом затухания $\Gamma=1 / 2 \tau_{21}$.

При упругом рассеянии на статических дефектах

$$
\frac{1}{\tau_{21}(\omega)}=\frac{2 \pi}{\hbar^{2}} \frac{2}{3} N_{i} \sum_{k_{1}}\left|V\left(\mathbf{k}_{1}-\mathbf{k}_{2}\right)\right|^{2} \delta\left(\omega-\omega_{1 \mathbf{k}_{1}}^{(T)}\right)
$$

где $N_{i}$ - концентрация дефектов, $V(\mathbf{q})-$ фурье-образ возмущающего потенциала. Для изотропного потенциала $V(\mathbf{q}) \equiv V(q)$. Заметим, что при $\omega_{M} \ll \omega_{L T} / \varepsilon_{b}$ величина $k_{2}(\omega)$ мала по сравнению с $k_{1}(\omega)$ и в $(9 \mathrm{c}) V\left(\mathbf{k}_{1}-\mathbf{k}_{2}\right)$ можно заменить на $V\left(\mathbf{k}_{1}\right)$. 
В случае излучения с $p$-поляризацией для расчета парциальных вкладов $I_{1 p}^{(0)}, I_{3 p}^{(0)}$ и интерференционной составляющей $I_{p 23}^{(0)}$ в суммарную интенсивность $I_{p}^{(0)}$ по (8):

$$
I_{p}^{(0)}(\omega, \Omega)=\sum_{\beta=1,2,3} I_{\beta p}^{(0)}(\omega, \Omega)+I_{p 23}^{(0)}(\omega, \Omega)
$$

рассмотрим функции Грина $G_{2 p}^{-+}\left(\mathbf{k}, \mathbf{k}^{\prime} ; \omega\right)$ для поперечных поляритонов ветви 2 и $G_{3 p}^{-+}\left(\mathbf{k}, \mathbf{k}^{\prime} ; \omega\right)$ для продольных экситонов. Диаграмма для этих функций изображена на рис. 1 , где $\lambda, \lambda^{\prime}=p$ и $\beta, \beta^{\prime}=2,3$. Сплошной линии в случае продольного экситона отвечает функция Грина

$$
\begin{aligned}
G_{3 \mathbf{k} \omega}^{--} & =\left[\omega-\omega_{k}^{(3)}+i \Gamma(\omega, \mathbf{k}) / 2\right]^{-1} \\
& =-\frac{2 M}{\hbar} \frac{1}{k^{2}-k_{3}^{2}}
\end{aligned}
$$

где $\omega_{\mathbf{k}}^{(3)}-$ определяется формулой $(6)$, а $k_{3}$ является решением дисперсионного уравнения продольных экситонов:

$$
k_{3}=n_{3} k_{0}, \quad n_{3}=\left(\left(\omega+i \frac{\Gamma}{2}-\omega_{L}\right) / \omega_{M}\right)^{1 / 2} .
$$

Используя рассуждения и технику выкладки для $s$ поляризации, получим

$$
\begin{gathered}
I_{\beta p}^{(0)}(\omega, \theta)=F_{\beta} \frac{\left|F_{\beta p}\right|^{2}}{2 k_{0} \operatorname{Im} n_{\beta z}+L^{-1}}, \beta=2,3, \\
I_{p 23}^{(0)}(\omega, \theta)=2 \operatorname{Re}\left[F_{23} \frac{F_{2 p} F_{3 p}^{*}}{-i k_{0}\left(n_{2 z}-n_{3 z}^{*}\right)+L^{-1}}\right],
\end{gathered}
$$

где

$$
\begin{gathered}
F_{\beta}=\frac{1}{(2 \pi)^{3}} \frac{2 M^{2} c \varepsilon_{b} \omega_{L T} \cos \theta f_{1}(\omega)}{\hbar \tau_{\beta 1}}, \\
F_{2 p}=\frac{t_{02}^{(p)}}{n_{2 z}\left(n_{1}^{2}-n_{2}^{2}\right)}, \quad F_{3 p}=\frac{t_{03}^{(p)}}{n_{3 z} \varepsilon_{b}},
\end{gathered}
$$

a $F_{23}$ получается из $F_{\beta}$ заменой величины $\tau_{\beta 1}$ на $\left(\tau_{21} \tau_{31}\right)^{1 / 2}$. При этом учитывалось, что для продольных экситонов напряженность электрического поля и экситонная часть поляризации кристалла связаны соотношением $4 \pi \mathbf{P}_{3}=-\varepsilon_{b} \mathbf{E}_{3}$. Выражение (14) описывает интерференционный вклад волн ветвей 2 и 3 в интенсивность внешнего излучения $p$-поляризации, обусловленный рассеянием на одном и том же центре поляритонов ветви 1 в когерентно излучающие состояния 2 и 3 в условиях, когда наряду с (1) нарушается и неравенство (2).

Теперь остается рассчитать амплитудные коэффициенты пропускания $t_{0 \beta}^{(p)}$ волны $\beta$ в вакуум. При этом использовали наряду с граничными условиями Максвелла еще и дополнительное граничное условие Пекара с „мертвым слоем“ [7]. В случае падения изнутри кристалла на его поверхность волны 2 с $p$-поляризацией находим

$$
\begin{aligned}
& t_{02}^{(p)}(\theta)=\frac{n_{1}^{2}-n_{2}^{2}}{n_{1}^{2}-\varepsilon_{b}} \\
& \times \frac{2 n_{2 z} / n_{2}}{\left(n_{0 z}+\bar{n}_{p}\right) \cos \delta-i \sin \delta\left[\frac{\tilde{n}_{z}}{\varepsilon_{b}}\left(1+\bar{n}_{p 1}\right)+\frac{n_{0 z} \varepsilon_{b}}{\tilde{n}_{z}} \bar{n}_{p 2}\right]},
\end{aligned}
$$

где

$$
\begin{gathered}
\bar{n}_{p}=n_{0 z} \bar{n}_{p 1}+\bar{n}_{p 2}, \quad \bar{n}_{p 2}=\left(n_{2 z}+n_{1 z} \bar{n}_{p 1}\right) \varepsilon_{b}, \\
\bar{n}_{p 1}=\frac{n_{1}^{2} n_{2 z} n_{3 z}+n_{0 x}^{2}}{n_{2}^{2} n_{1 z} n_{3 z}+n_{0 x}^{2}} \frac{\varepsilon_{b}-n_{2}^{2}}{n_{1}^{2}-\varepsilon_{b}}, \quad n_{\beta x}=n_{0 x}=\sin \theta .
\end{gathered}
$$

Коэффициент пропускания $t_{01}^{(p)}$ получается из $t_{02}^{(p)}$ симметричной заменой $n_{1} \leftrightarrow n_{2}$. А в случае падения изнутри кристалла на его поверхность продольной волны определяем амплитудный коэффициент превращения нормальной волны продольных экситонов во внешнее световое излучение в следующем виде:

$$
\begin{aligned}
& t_{03}^{(p)}(\theta)= \frac{2 n_{0 x}\left(n_{1 z}-n_{2 z}\right) n_{3 z} / n_{3}}{\sum_{\beta=1,2}(-1)^{\beta} \frac{n_{\beta}^{2}-\varepsilon_{\beta}}{n_{\beta}^{2}}\left(n_{0 x}^{2}+n_{\beta z} n_{3 z}\right)\left[\left(n_{0 z}\right.\right.} . \\
&\left.\left.\quad+\frac{n_{3-\beta, z}}{\varepsilon_{b}}\right) \cos \delta-i \sin \delta\left(\frac{\tilde{n}_{z}}{\varepsilon_{b}}+\frac{n_{0 z} n_{3-\beta, z}}{\tilde{n}_{z}}\right)\right]
\end{aligned}
$$

\section{Результаты численного расчета и сравнение с экспериментом}

Для численного расчета парциальных $I_{\beta p}^{(0)}(\omega), I_{\beta s}^{(0)}$ и интегральных $I_{s}^{(0)}(\omega), I_{p}^{(0)}$ спектральных интенсивностей люминесценции воспользовались формулами (8)-(10) и (13)-(15) как основными, а также вспомогательными формулами типа (16), (17) для амплитудных коэффициентов пропускания $t_{0 \beta}^{(\lambda)}$ и показателей преломления $n_{\beta}$. Единственным варьируемым параметром теории является эффективная глубина распределения поляритонов $L$ нижней ветви 1. Значение $\hbar \Gamma$ считаем известным из эксперимента. Численные расчеты проводились при следующих значениях основных параметров экситонного резонанса $A_{n=1}$ кристалла $\mathrm{CdS}$ : $\hbar \omega_{0}=2.5524 \mathrm{eV}$, $\hbar \omega_{L T}=2.0 \mathrm{meV}, M_{e x}=0.9 m_{0} \quad\left(m_{0}\right.$ - масса свободного электрона), фоновая диэлектрическая проницаемость $\varepsilon_{b}=9.4$, толщина „мертвого слоя“ $\lambda=70^{\circ}$. Эти значения параметров хорошо согласуются с экспериментальными результатами по экситонному отражению света и экситонной люминесценции при $T=2 \mathrm{~K}[4,7]$.

На рис. 2 приведены теоретические спектральные зависимости $I_{p}^{(0)}(a), I_{2 p}^{(0)}(b), I_{3 p}^{(0)}(c)$ и $I_{p 23}^{(0)}(d)$, рассчитанные при $\theta=80^{\circ}$ и разных значениях параметров $\Gamma$ и $L$. Оказалось, что интенсивность и полуширина кривых $I_{2 p}^{(0)}(\omega)$, и следовательно, $I_{p}^{(0)}(\omega)$ существенно зависят от $\Gamma L$. Аналогичные параметры кривых $I_{3 p}^{(0)}(\omega)$ и $I_{p 23}^{(0)}(\omega)$ при $L>1 \mu \mathrm{m}$ практически не чувствуют изменение значения $L$, что обусловлено относительно 

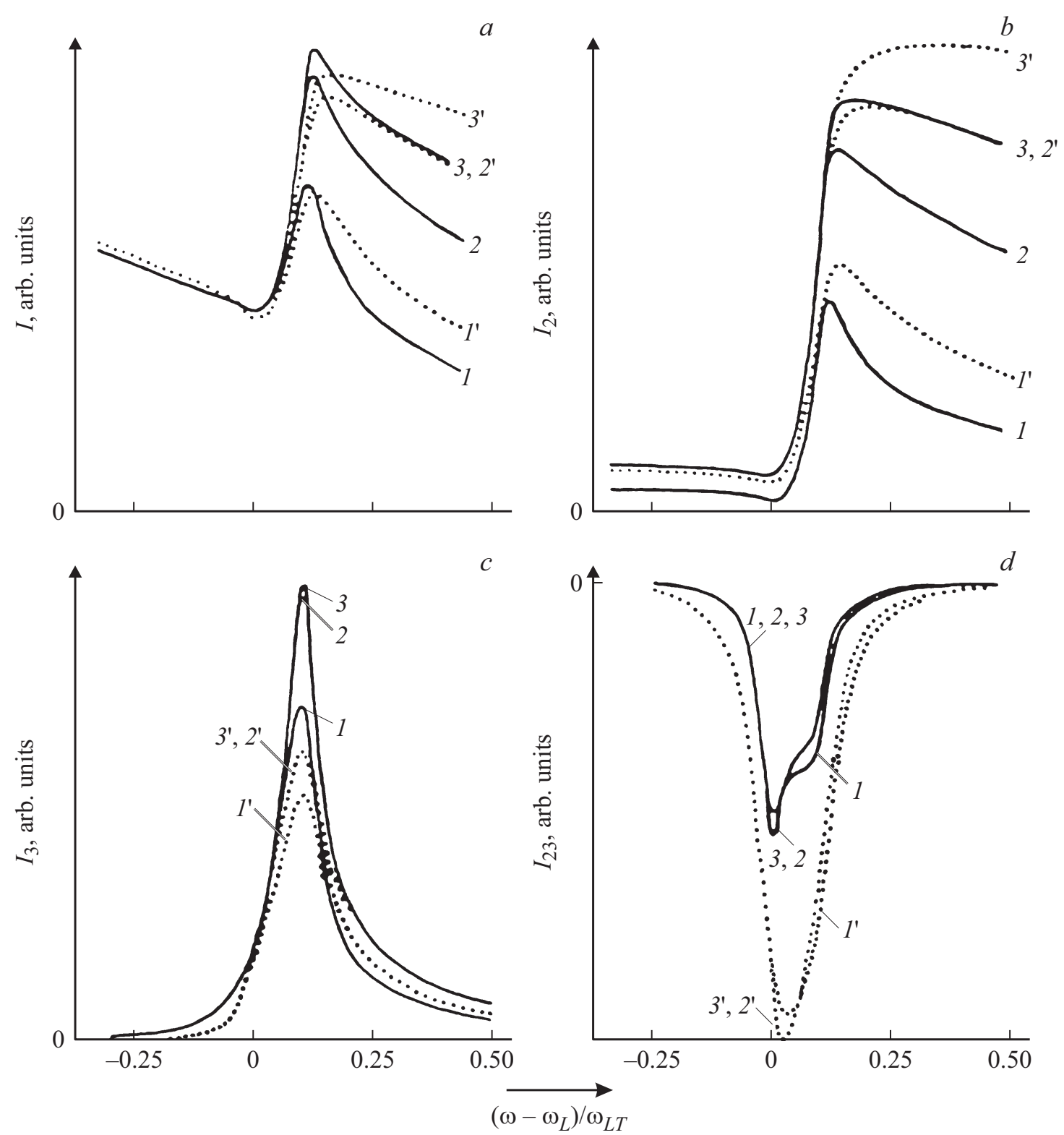

Рис. 2. Спектральные зависимости полной $I_{p}^{(0)}$, парциальных $I_{2 p}^{(0)}, I_{3 p}^{(0)}$ интенсивностей и интерференционного вклада $I_{23 p}^{(0)}$, рассчитанные для параметров экситонного резонанса $A_{(n=1)}$ в $\mathrm{CdS}$ при угле выхода в вакуум $\theta=80^{\circ}$ и для разных значений экситонного затухания $\hbar \Gamma=0.075(1-3), 0.15 \mathrm{meV}\left(1^{\prime}-3^{\prime}\right)$ и глубины распределения поляритонов ветви $1: L=0.2\left(1,1^{\prime}\right)$, $1.0\left(2,2^{\prime}\right), 2.0 \mu \mathrm{m}\left(3,3^{\prime}\right)$.

большим значением коэффициента поглощения волны 3 $\left(\alpha_{3} L \gg 1\right)$. Более того, полуширина $\Delta_{3}$ кривой $I_{3 p}^{(0)}(\omega)$ почти полностью определяется величиной Г. Согласно численному расчету $\Delta_{3} \approx 2 \Gamma$. Заметим, что вклад $I_{3 p}^{(0)}$ в $I_{p}^{(0)}$ с ростом $\hbar \Gamma$ резко уменьшается, при этом $I_{2 p}^{(0)}-$ растет и сильно уширяется, в результате чего при одном и том же значении $L$ увеличение $\Gamma$ приводит к слабому уменьшению максимума $I_{p}^{(0)}(\omega)$ на частоте $\omega_{\theta}$ и его уширению. Это объясняется результатом подавления ПД для продольных волн затуханием механических экситонов.

Квантовую теорию, развитую выше, используем для описания экспериментальных спектров ЭПЛ кристаллов
$\mathrm{CdS}$ в „изотропных“ геометриях излучения $(s-$ поляризация, когда $\Omega(\theta, \varphi)$ лежит в плоскости $Y Z$ и оптическая ось $C \| \mathrm{Y} ; p-$ поляризация, когда $\Omega \perp C \| X)$. Спектры регистрировались на установке профессора Селькина А.В., собранной на базе спектрометра ДФС-24, в режиме счета фотонов при минимальной спектральной ширине щели $0.04 \mathrm{meV}$ в условиях максимально возможного спектрального разрешения. Возбуждение ЭПЛ осуществлялось на длине волны $\lambda=476.5 \mathrm{~nm}$ светом $\mathrm{Ar}^{+}$лазера, сфокусированным на поверхность кристалла в пятно размером $\sim 0.4 \times 4 \mathrm{~mm}$ при мощности светового потока $\sim 7 \mathrm{~mW}$. 

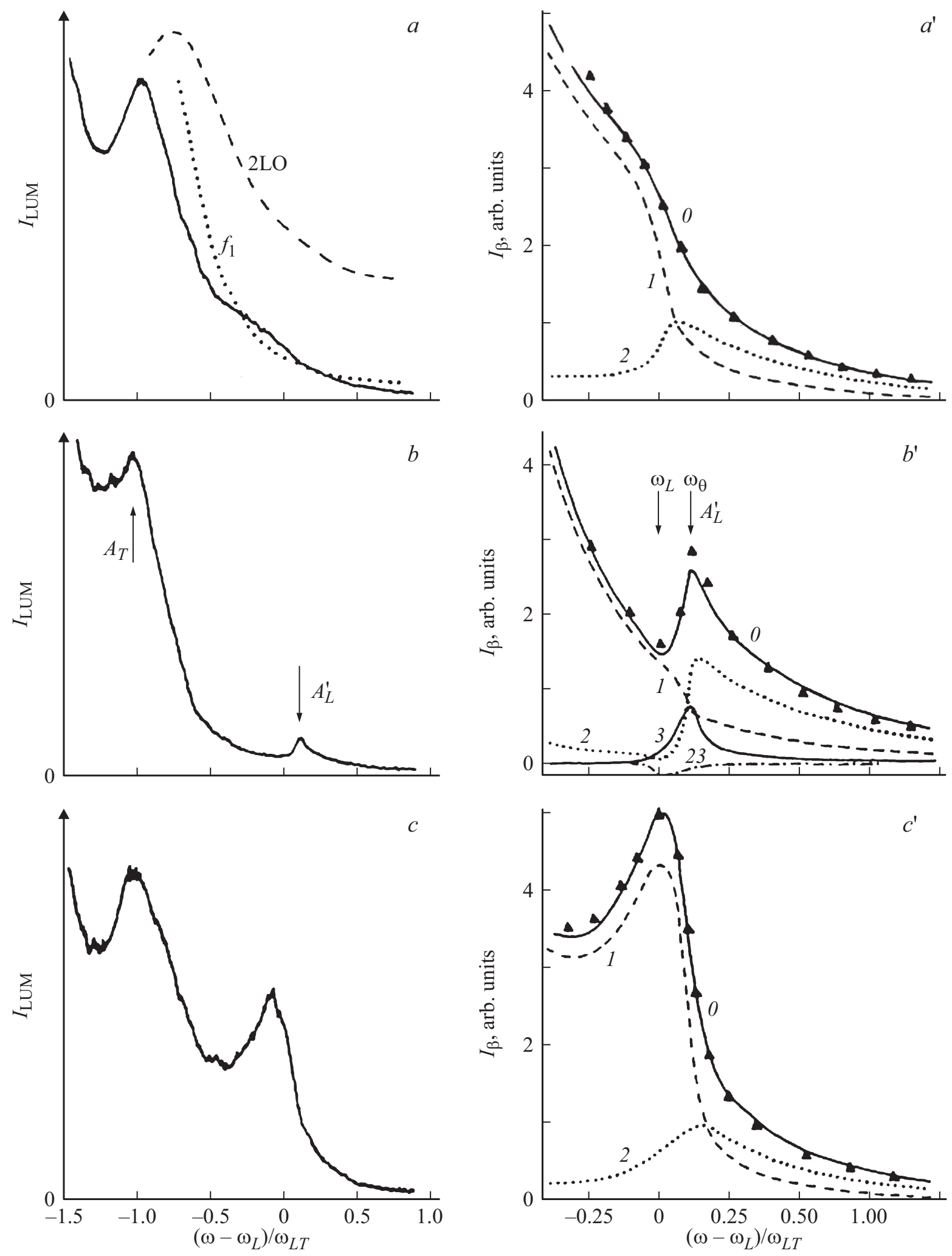

Рис. 3. Экспериментальные $(a, b, c)$ и теоретические $\left(a^{\prime}, b^{\prime}, c^{\prime}\right)$ спектры ЭПЛ кристаллов $\mathrm{CdS}(T=2 \mathrm{~K})$ при $s$ - $\left(a, a^{\prime} ; c, c^{\prime}\right)$ и $p$-поляризации $\left(b, b^{\prime}\right)$ для углов выхода $\theta=0\left(a, a^{\prime}\right)$ и $80^{\circ}\left(b, b^{\prime}, c, c^{\prime}\right) .0-$ суммарная интенсивность излучения, треугольники эксперимент; 1, 2 и 3 - вклады поперечных поляритонов (ветви 1,2) и продольных экситонов (ветвь 3 ), 23 - интерференционный вклад $I_{p 23}^{(0)}$. 
На рис. 3, a, $c$ представлены общий вид контуров люминесценции кристалла $\mathrm{CdS}\left(A_{n=1}, T=2 \mathrm{~K}\right)$ в $s$-геометрии излучения при $\theta=0$ (a) и $\theta=80^{\circ}(c)$. Штриховая линия на рис. 3, $a$ изображает экспериментально измеренную по спектру 2LO-повторения функцию заселенности $g_{1}(\omega)=\rho_{1}(\omega) f_{1}(\omega)$ поляритонных состояний ветви 1, откуда определялась функция распределения $f_{1}(\omega)$, показанная там же пунктирной линией. Теоретические спектры ЭПЛ на рис. $3, a^{\prime}, c^{\prime}$ (кривые 0), рассчитанные при значениях параметров $\hbar \Gamma=0.075 \mathrm{meV}, L=0.8 \mu \mathrm{m}$ и $\delta=70^{\circ}$, хорошо совпадают с экспериментом (треугольники). Парциальный вклад $I_{2 s}^{(0)}$ (пунктирные кривые 2 ) играет существенную роль в формировании суммарной интенсивности $I_{s}^{(0)}$ в области частот $\omega \geq \omega_{L}$ и даже превосходит вклад $I_{1 s}^{(0)}$ (штриховые кривые 1 ).

На рис. 3, $b$ изображен экспериментальный контур ЭПЛ $\mathrm{CdS}$ при р-поляризации и $\theta \approx 80^{\circ}$, в которой наблюдается наряду с основным максимумом излучения $A_{T}$ еще и дополнительный небольшой максимум $A_{L}^{\prime}$, расположенный с коротковолновой стороны от $\omega_{L}$ на частоте $\omega_{\theta=80^{\circ}}=2.5547 \mathrm{eV} / \hbar$. Появление максимума $A_{L}^{\prime}$ ранее объяснялось только „высвечиванием“ чисто продольных экситонов. Однако, как показывает результаты численного расчета, представленные на рис. $3, b^{\prime}$, линия $A_{L}^{\prime}$ формируется сложным образом. Спектральные зависимости суммарной интенсивности $I_{p}^{(0)}$ (сплошная кривая 0), парциальных вкладов $I_{1 p}^{(0)}$ (кривая 1$), I_{2 p}^{(0)}$ (кривая 2), $I_{3 p}^{(0)}$ (кривая 3) и интерференционной составляющей $I_{p 23}^{(0)}($ кривая 23) рассчитывались для известных параметров CdS [2] и при тех же значениях $\Gamma, L, l$, что и кривые на рис. $3, a^{\prime}, c^{\prime}$.

Обращает на себя внимание область частот $\omega<\omega_{\theta}$, где с точки зрения кинетического приближения продольные экситоны так же, как и поперечные поляритоны 2, не могут превращаться во внешние фотоны, т. е. высвечиваться в вакуум. Согласно квантовой теории, совпадающей с экспериментом (cp. кривую $O$ и треугольники на рис. $\left.3, b^{\prime}\right)$, экситонное затухание индуцирует излучение поверхностно-радиационных мод 2 и 3, а также их интерференцию. Это, несомненно, является новым механизмом формирования ЭПЛ в кристаллах с пространственной дисперсией. Строго говоря, частоты $\omega_{\theta}$ для волны 2 и 3 несколько отличаются. Поскольку при $\Gamma=0$ в окрестности $\omega_{L}$ дисперсионные зависимости волн 2 и 3 можно выразить с точностью до малого параметра $\omega_{M} \varepsilon_{b} / \omega_{L T} \ll 1$ формулами

$$
\begin{gathered}
\omega_{2 \mathbf{k}}^{(T)} \approx \omega_{L}+n_{2}^{2}(\omega) \omega_{L T} / \varepsilon_{b}, \\
\omega_{3 \mathbf{k}}^{(L)} \approx \omega_{L}+n_{3}^{2}(\omega) \omega_{M},
\end{gathered}
$$

то находим из (18) и (19) следующие соотношения для характерных частот $\omega_{\theta}^{(2)}$ и $\omega_{\theta}^{(3)}$ :

$$
\omega_{\theta}^{(2)} \approx \omega_{L}+\sin ^{2} \theta \omega_{L T} / \varepsilon_{b},
$$

$$
\omega_{\theta}^{(3)} \approx \omega_{L}+\sin \theta \omega_{M}
$$

В случае предельно больших углов выхода $\left(\theta \approx 85^{\circ}\right)$ отсюда получим для параметров $\mathrm{CdS}(T=2 \mathrm{~K})$

$$
\begin{gathered}
\omega_{\theta, \max }^{(2)} \approx \omega_{L}+\omega_{L T} / \varepsilon_{b}, \quad \omega_{L T} / \varepsilon_{b} \approx 0.22 \mathrm{meV}, \\
\omega_{\theta, \max }^{(3)} \approx \omega_{L}+\omega_{M}, \hbar \omega_{M} \cong 7 \cdot 10^{-3} \mathrm{meV}, \\
\hbar \omega_{L}=2.5544 \mathrm{eV} .
\end{gathered}
$$

Согласно эксперименту и численному расчету (рис. $3, b^{\prime}$ ), максимум линии излучения $A_{L}^{\prime}$ находится вблизи характерной частоты поперечного поляритона 2: $\hbar \omega_{\theta, \max }^{(2)} \approx 2.5546 \mathrm{eV}$. Из-за влияния экситонного затухания $\Gamma$ реальный максимум частотной зависимости $I_{3 p}^{(0)}$ смещается в коротковолновую сторону от частоты $\omega_{L}$ и приближается к частоте $\omega_{\theta} \approx \omega_{\theta}^{(2)}$. Рис. $3, b^{\prime}$ также показывает, что контур линии $A_{L}^{\prime}$ нельзя получить, ограничиваясь лишь вкладом $I_{3 p}^{(0)}$. На частоте $\omega_{\theta}$ отношения $I_{1 p}^{(0)}, I_{2 p}^{(0)}, I_{3 p}^{(0)}$ и $I_{p 23}^{(0)}$ к суммарной $I_{p}^{(0)}$ составляют $0.30,0.52,0.30,-0.12$ соответственно. Особо следует отметить, что даже для практически минимального значения $\hbar \Gamma=0.075 \mathrm{meV}$ в кристаллах $\mathrm{CdS}$ неприменимо кинетическое приближение для описания парциальных вкладов $I_{2 p}^{(0)}$ и $I_{3 p}^{(0)}$ в окрестности линии излучения $A_{L}^{\prime}$, так как $\left(\omega_{\theta, \max }-\omega_{L}\right) / \Gamma \approx 2.0$ и неравенство (1) на частоте $\omega=\omega_{\theta}$ не выполняется для продольной волны, а для поперечной моды $\omega_{\theta}$ является критической частотой, ниже которой для данного направления $\Omega(\theta, \varphi)$ в вакууме величина $\mathbf{k}_{2}$ является чисто мнимой при $\Gamma=0$.

На рис. 4 сравниваются теоретические (кривые 0 ) и экспериментальные (треугольники) спектры ЭПЛ кристаллов $\mathrm{CdS}$, а также расчетные частотные зависимости парциальных вкладов (кривые $1,2,3,23)$, соответствующие $s$ - $(a, c, e)$ и $p$-поляризациям $(b, d, f)$ при разных значениях угла выхода в вакуум $\theta: 30^{\circ}(a, b), 45^{\circ}(c, d)$ и $60^{\circ}(e, f)$. Видно, что относительные вклады $I_{1 \lambda}^{(0)}$ и $I_{2 \lambda}^{(0)}$ в люминесценцию слабо зависят от $\theta$, однако вклад $I_{3 p}^{(0)}$ (кривые 3) нормальной волны 3 существенно увеличивается с ростом $\theta$ и играет основную роль в формировании линии $A_{L}^{\prime}$.

Рост вклада $I_{3 p}^{(0)}$ с увеличением $\theta$ непосредственно связан с проявлением ПД в спектрах люминесценции. Без ПД волны 3 так же, как и волны 1, не могут существовать в области частот $\omega>\omega_{L}$. При $\theta=0$ продольные волны 3 испытывают полное внутреннее отражение и поэтому вклад $I_{3 p}^{(0)}$ в $I_{p}^{(0)}$ отсутствует. Последнее имеет место также при $n_{3 L}>\sin \theta$, чем и объясняется резкое уменьшение $I_{3 p}^{(0)}$ с ростом частоты в области $\omega>\omega_{\theta}$.

Интересно заметить, что интерференционная составляющая $I_{p 23}^{(0)}$ дает незначительный отрицательный вклад $(\sim 10 \%)$ в суммарную интенсивность $I_{p}^{(0)}$, абсолютное значение которого уменьшается с увеличением $\theta$, когда происходит „расталкивание“ мод 2 и 3. Однако без 

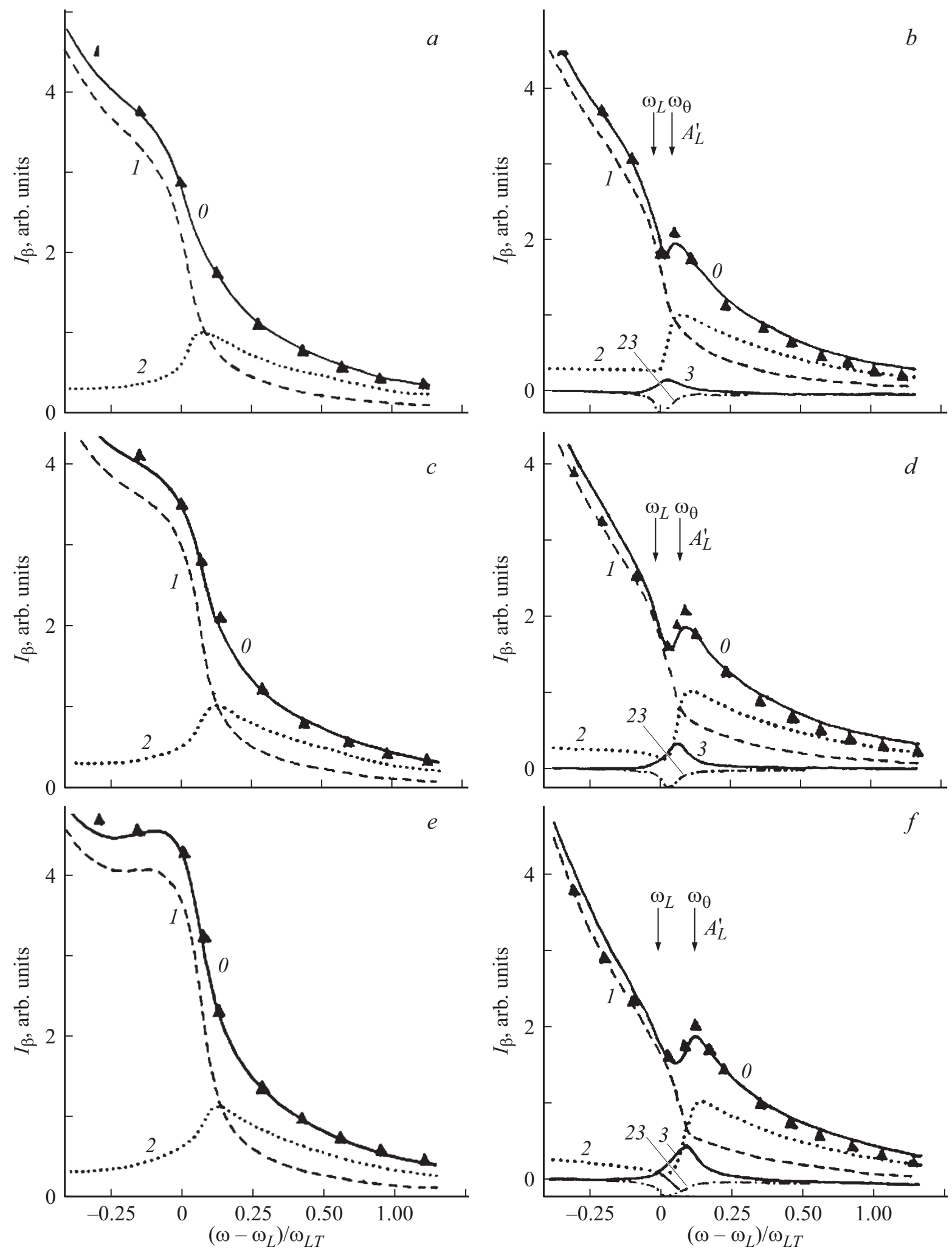

Рис. 4. Сравнение теоретических $(0)$ и экспериментальных (треугольники) спектров ЭПЛ при $s$ - $(a, c, e)$ и $p$-поляризации $(b, d, f)$ излучения кристаллов $\mathrm{CdS}$ для углов выхода $\theta=30^{\circ}(a, b), 45^{\circ}(c, d), 60^{\circ}(e, f)$. Обозначения такие же, как и на рис. 3. 
вклада $I_{p 23}^{(0)}$ количественно трудно получить небольшой минимум в спектре ЭПЛ на частоте вблизи $\omega_{L}$, практически не зависящий от $\theta$. Смещение линии $A_{L}^{\prime}$ с ростом угла $\theta$ в коротковолновую сторону от $\omega_{L}$ при $\omega_{M} \varepsilon_{b} \ll \omega_{L T}$ и $\Gamma \ll\left(\omega_{\theta}-\omega_{L}\right)$ проанализируем более подробно. В таком случае, учитывая, что $n_{\beta}^{2}=n_{\beta z}^{2}+\sin ^{2} \theta$, дисперсионное соотношение для излучающих в вакуум состояний ветви 2 (вблизи $\omega_{L}$ ) можем записать как

$$
n_{2 z}^{2} \cong \varepsilon_{b}-\frac{\varepsilon_{b} \omega_{L T}^{\prime}}{\omega-\omega_{0}},
$$

где

$$
\omega_{L T}^{\prime}=\omega_{L T}+\frac{\omega-\omega_{0}}{\varepsilon_{b}} \sin ^{2} \theta \approx \omega_{L T}\left(1+\frac{\sin ^{2} \theta}{\varepsilon_{b}}\right) .
$$

Естественно, что для значений $\theta$, при которых $n_{2 z}^{2}<0$, волна 2 испытывает пространственное затухание даже при $\Gamma=0$. При $\theta=0$ это происходит, как видно из $(22)$, в области частот $\omega<\omega_{L}$, а при $\theta \neq 0-$ в области частот ниже $\omega_{\theta}$, для которой из $(22)$ получим формулу

$$
\omega_{\theta}=\omega_{L}+\left(\frac{\sin ^{2} \theta}{\varepsilon_{b}-\sin ^{2} \theta} \omega_{L T}\right),
$$

отличающуюся от (20) лишь заменой $\varepsilon_{b}$ на $\varepsilon_{b}-\sin ^{2} \theta$. Таким образом, с ростом $\theta$ дно энергетической зоны излучающих в вакуум „легких“ поперечных поляритонов смещается вверх, т. е. происходит некоторое увеличение продольно-поперечного расщепления $\omega_{L T}$ (23). Этот эффект, в основном, и проявляется в смещении линии излучения $A_{L}^{\prime}$ с ростом $\theta$. Для двух значений $\theta_{1}$ и $\theta_{2}$ угла выхода из (24) находим отношение

$$
\frac{\omega_{\theta 1}-\omega_{L}}{\omega_{\theta 2}-\omega_{L}}=\frac{\left(\varepsilon_{b} / \sin ^{2} \theta_{2}\right)-1}{\left(\varepsilon_{b} / \sin ^{2} \theta_{1}\right)-1}
$$

из которого при $\theta_{1}=60^{\circ}, \theta_{2}=30^{\circ}$, для $\operatorname{CdS}\left(\varepsilon_{b}=9.4\right)$ получим значение 3.2 , тогда как рис. $4, f$ дает несколько другое значение, равное 2.8. Такое небольшое несоответствие объяснятся тем, что условие $\Gamma \ll\left(\omega_{\theta}-\omega_{L}\right)$ в данном случае не совсем выполняется.

\section{Заключение}

Развита теория экситон-поляритонной люминесценции кубических кристаллов с пространственной дисперсией для продольных экситонов в условиях нарушения критериев применимости кинетического уравнения Больцмана.

Процесс формирования спектра ЭПЛ имеет ряд специфических особенностей: перестройка энергетического спектра нормальных волн с учетом реального экситонного затухания приводит к включению в перенос энергии через границу кристалла поверхностнорадиационных мод, а наличие ПД - к их интерференционному взаимодействию; вклады $I_{3 p}^{(0)}, I_{23 p}^{(0)}$ с ростом $ћ \Gamma$ резко уменьшаются, что объясняется результатом подавления ПД затуханием механических экситонов для продольных волн, тогда как вклад $I_{2 p}^{(0)}-$ растет и сильно уширяется, в результате чего увеличение $Г$ приводит к слабому уменьшению максимума $I_{p}^{(0)}(\omega)$ на частоте $\omega_{\theta}$ и его уширению.

Вклад в ЭПЛ чисто продольных экситонов и их интерференции с поляритонами верхней дисперсионной ветви небольшие $(\sim 10-30 \%)$, тем не менее, учет их необходим для правильного количественного описания эксперимента.

Изложенный метод анализа спектров ЭПЛ дает возможность определить оптические параметры кристалла (например, значение $\omega_{L T}$ по частоте $A_{L}^{\prime}$-линии) и может быть использован с небольшими изменениями при изучении спектров неупругих рассеяний поляритонов в окрестности частоты продольного экситона с учетом конечного затухания и ПД.

В заключение авторы выражают искреннею признательность А.В. Селькину за представленные экспериментальные спектры ЭПЛ.

\section{Список литературы}

[1] Benoit a la Guillame C., Bonnot A., Delever J.M. // Phys. Rev. Lett. 1970. V. 24. P. 1235.

[2] Cross E.F., Permogorov S.A., Travnikov V.V., Sel'kin A.V. // Sol. State Commun. 1972. V. 10. P. 1071.

[3] Пермогоров С.А., Травников В.В., Селькин А.В. // ФТТ. 1972. T. 14. № 2. C. 3642.

[4] Sel'kin A.V. // Phys. Stat. Sol. B. 1977. V. 83. P. 47.

[5] Ивченко Е.Л., Пикус Г.Е., Юлдашев Н.Х. // ЖЭТФ. 1981. T. 80. № 3. C. 1228.

[6] Абдукадыров А.Г., Сажин М.И., Селькин А.В., Юлдашев H.X. // Опт. и спектр. 1989. Т. 67. № 10. С. 845.

[7] Абдукадыров А.Г., Ивченко Е.Л., Сажин М.И., Селькин А.В., Юлдашев Н.Х. // ЖЭТФ. 1990. Т. 97. С. 644.

[8] Юлдашев Н.X. Экситон-поляритонная люминесценция и перенос резонансного излучения в кристаллах. Фергана: Фаргона, 2001. 214 c.

[9] Ахмадалиев Б.Ж., Полвонов Б.З., Юлдашев Н.Х. // Опт. и спектр. 2014. Т. 116. С. 106; Akhmadaliev B.J., Polvonov B.Z., Yuldashev N. Kh. // Opt. Spectrosc. 2014. V. 116. P. 244.

[10] Пермогоров С.А., Суркова Т.П., Тенищев А.Н. // ФТТ. 1998. T. 40. C. 897.

[11] Багаев В.С., Клевков Ю.В., Колосов С.А., Кривобок В.С., Шепель А.А. // ФТТ. 2010. Т. 52. С. 37. 